\title{
Chemical freeze-out systematics of thermal model analysis using hadron yield ratios
}

\author{
Sumana Bhattacharyya $\odot,{ }^{1, *}$ Amaresh Jaiswal $\odot,{ }^{2, \dagger}$ and Sutanu Roy ${ }^{3, \dagger}$ \\ ${ }^{1}$ Department of Physics, Center for Astroparticle Physics \& Space Science, Bose Institute, Kolkata 700091, India \\ ${ }^{2}$ School of Physical Sciences, National Institute of Science Education and Research, HBNI, Jatni 752050, Odisha, India \\ ${ }^{3}$ School of Mathematical Sciences, National Institute of Science Education and Research, HBNI, Jatni 752050, Odisha, India
}

(Received 5 October 2020; accepted 19 January 2021; published 5 February 2021)

\begin{abstract}
We provide a framework to estimate the systematic uncertainties in chemical freeze-out parameters extracted from $\chi^{2}$ analysis of thermal models, using hadron multiplicity ratios in relativistic heavy-ion collision experiments. Using a well known technique of graph theory, we construct all possible sets of independent ratios from available hadron yields and perform $\chi^{2}$ minimization on each set. We show that even for ten hadron yields, one obtains a large number $\left(10^{8}\right)$ of independent sets which results in a distribution of extracted freeze-out parameters. We analyze these distributions and compare our results for chemical freeze-out parameters and associated systematic uncertainties with previous results available in the literature.
\end{abstract}

DOI: 10.1103/PhysRevC.103.024905

\section{INTRODUCTION}

Relativistic heavy-ion collisions provides the opportunity to create hot and dense QCD matter and study its thermodynamic and transport properties. The "standard" model of relativistic heavy-ion collision has been developed in last few decades by analyzing the experimental data from Relativistic Heavy-Ion Collider (RHIC) at Brookhaven National Lab, USA and the Large Hadron Collider (LHC) at CERN in Geneva, Switzerland. The analysis of experimental data suggests that the fireball produced in these collisions consists of deconfined quarks and gluons in the early stage of evolution which tends to thermalize rapidly. This quark-gluon plasma (QGP) undergoes a transition from partonic phase to hadronic phase during the later stage of evolution and finally the energy-momenta of these hadrons are measured by the detectors. The rapid thermalization of the fireball, due to strongly interacting constituents of the medium, provides the motivation to look for thermal description of the observed hadron yields. Thermal models of the hadronic system have a long history [1-5]. Indeed, statistical models, with the assumption of complete thermalization of hadronic matter, have been quite successful in explaining the hadron yields and their ratios measured in relatively recent experiments [6-42].

Thermodynamic parameters obtained from statistical model analysis can be used to characterize the freeze-out

\footnotetext{
*response2sumana91@gmail.com

†a.jaiswal@niser.ac.in

‡sutanu@niser.ac.in
}

Published by the American Physical Society under the terms of the Creative Commons Attribution 4.0 International license. Further distribution of this work must maintain attribution to the author $(s)$ and the published article's title, journal citation, and DOI. Funded by $S C O A P^{3}$. hypersurface as the last surface of interaction. The onset of chemical freeze-out is said to have occurred when particle abundances are fixed and composition altering inelastic interactions have ceased. Subsequently kinetic freeze-out occurs when elastic interactions between particles have also stopped and the density drops to the level at which the hadron momentum spectra remain unchanged. Beyond kinetic freeze-out, hadrons are assumed to stream freely to the detector. The hadron resonance gas (HRG) model, which assumes a statistical description of a mixture of hadrons and their resonances in thermodynamic equilibrium, leads to a good description of the medium at freeze-out, for a wide range of collision energy [6-44]. In this model, the thermodynamic equilibrium state of the strongly interacting matter is completely determined by the temperature $(T)$ and the three chemical potentials $\mu_{Q}, \mu_{B}$, and $\mu_{S}$ corresponding to baryon number $(B)$, electric charge $(Q)$, and strangeness $(S)$, respectively. These parameters at freeze-out can be extracted from statistical model calculations by performing a $\chi^{2}$ minimization fit to the available experimental multiplicity data [21-24,45-51]. Several codes, such as THERMUS [52], SHARE [53], and THERMINATOR [54], are publicly available to compute the abundances of particles using such a statistical hadronization approach.

The systematic uncertainties in the experimental data are expected to be reduced when one considers the ratios of hadron yields for $\chi^{2}$ analysis, which overall cancels the the system volume $(V)[21,55]$. However, there are multiple ways to form a set of $(N-1)$ independent ratios given $N$ number of hadron yields. For simplicity, we call a set containing $(N-1)$ independent ratios an independent set. It is evident that the choice of particle ratios may introduce a bias for the extracted freeze-out parameters $[21,56]$. Therefore, the freeze-out parameters extracted from a $\chi^{2}$ minimization fit to these ratios would depend on the set under consideration. To avoid this problem, one may try to fit the absolute yields rather than their ratios, which requires the inclusion of an additional parameter 
$V$. This, however, is subject to its own bias [21,22,24]. It has also been observed that fitting absolute yield is more prone to converge to a false minimum because of the strong correlation between yield normalization and other freeze-out parameters [53]. This problem becomes more pronounced when one tries to incorporate excluded volume effect [21]. On the other hand, yields ratios are not affected significantly when considering the excluded volume effect and therefore the extracted freezeout parameters remains stable.

In order to reliably extract freeze-out parameters from hadron yield ratios, one has to understand the systematic uncertainties due to the choice of specific ratios. The first attempt towards understanding this uncertainty was made recently in Ref. [56], where the authors considered several independent sets for extraction of freeze-out parameters. These sets were chosen such that each of the ${ }^{N} C_{2}$ ratios appear in at least one set. However, it is important to note that the choice of these particular sets is also not unique. Therefore, the bias arising from the choice of those specific sets still remains, which could only be removed by considering all possible independent sets. Given the importance of statistical models in the context of relativistic heavy-ion collisions, it is essential to conclusively address the issue of systematic uncertainty.

In this article, we provide a framework to estimate systematic uncertainties in the extraction of chemical freeze-out parameters from analysis of hadron multiplicity ratios. We construct all possible independent sets from available hadron yields by using a well known technique in graph theory. Subsequently, we perform $\chi^{2}$ minimization on each set, which leads to a distribution of the extracted freeze-out parameters. From these distributions, we obtain quantitative estimates of systematic uncertainty in the extracted freeze-out parameters corresponding to yield ratios of experimental data at $200 \mathrm{GeV}$ (RHIC) and 2.76 TeV (LHC) collision energies. We also estimate these uncertainties after removing the usual constraints on the conserved charges. Finally, we compare our results for chemical freeze-out parameters and associated systematic uncertainties with previous results available in the literature.

\section{HADRON RESONANCE GAS MODEL}

We consider the HRG model for our analysis of yield ratios. The thermodynamic potential of HRG in terms of the grand-canonical partition function is given by

$$
\ln Z^{\text {id }}= \pm \sum_{i} \frac{V g_{i}}{(2 \pi)^{3}} \int d^{3} p \ln \left[1 \pm \exp \left(-\frac{E_{i}-\mu_{i}}{T}\right)\right]
$$

where the sum runs over all hadrons and resonances. The upper sign is for fermions and lower is for bosons, and the normalization factor $V$ is the volume of the fireball. Here $g_{i}$, $E_{i}$, and $m_{i}$ are respectively the degeneracy factor, energy, and mass of the $i$ th hadron, while, $\mu_{i}=B_{i} \mu_{B}+S_{i} \mu_{S}+Q_{i} \mu_{Q}$ is the chemical potential of the $i$ th hadron with $B_{i}, S_{i}$, and $Q_{i}$ denoting its baryon number, strangeness, and electric charge.

For a thermalized system the number density $n_{i}$ obtained from partition function is given as

$$
n_{i}=\frac{T}{V}\left(\frac{\partial \ln Z_{i}}{\partial \mu_{i}}\right)_{V, T}=\frac{g_{i}}{(2 \pi)^{3}} \int \frac{d^{3} p}{\exp \left[\left(E_{i}-\mu_{i}\right) / T\right] \pm 1} .
$$

The rapidity density for $i$ th detected hadron corresponding to the number density in the HRG model can be written as [23]

$$
\left.\left.\frac{d N_{i}}{d y}\right|_{\text {Det }} \simeq \frac{d V}{d y} n_{i}^{\text {Tot }}\right|_{\text {Det }}
$$

where the subscript "Det" denotes the detected hadrons. If the heavier resonances $j$ decay to the $i$ th hadron, then

$$
n_{i}^{\text {Tot }}=n_{i}+\sum_{j} n_{j} \times \text { branching ratio of }(j \rightarrow i),
$$

where $n_{i}$ and $n_{j}$ depend on the thermal parameters $\left(T, \mu_{B}, \mu_{Q}, \mu_{S}\right)$, which can be obtained by fitting experimental hadron yields to the model calculations. The fitted values of thermal parameters are obtained by minimizing $\chi^{2}$, which is defined as

$$
\chi^{2}=\sum_{\alpha} \frac{\left(R_{\alpha}^{\exp }-R_{\alpha}^{\mathrm{th}}\right)^{2}}{\left(\sigma_{\alpha}^{\exp }\right)^{2}}
$$

where $R_{\alpha}^{\exp }$ is the ratio of hadron yields obtained from experiments, $R_{\alpha}^{\text {th }}$ is the ratio of the number densities from the model calculations, and $\sigma_{\alpha}^{\exp }$ is the experimental uncertainty.

As individual multiplicities or their ratios are not conserved by strong interactions, $\chi^{2}$ fitting is performed by choosing a set of multiplicity ratios. In general, with $N$ given experimental hadron yields $p_{1}, p_{2}, \ldots, p_{N}$ one can construct $N(N-1)$ ratios of the form $p_{i} / p_{j}$, where $1 \leqslant i, j \leqslant N$ and $i \neq j$. However, interchanging numerator and denominator does not provide any new information in our analysis. Therefore, it is sufficient to consider $N(N-1) / 2$ ratios of the given particle yields. Because of the correlation existing between all possible multiplicity ratios, it is only relevant to choose $(N-1)$ number of statistically independent ratios out of those $N(N-1) / 2$ ratios to parametrize the thermal model [21,57]. It has also been pointed out within a thermal model analysis that choosing a specific independent set may introduce bias in the minimization process [56]. However, it is possible to quantify this systematic uncertainty in the freeze-out parameters, extracted from $\chi^{2}$ fitting, by considering all possible sets of independent ratios. Observe that $(N-1)$ ratios can be chosen in ${ }^{N(N-1) / 2} C_{N-1}$ ways. But, any set containing $(N-1)$ ratios may not necessarily be independent. Also, enumerating all independent sets from ${ }^{N(N-1) / 2} C_{N-1}$ sets is a complex and tedious process because the total number of independent sets grows immensely for large $N$. We perform this enumeration process by relating it to a particular case of the minimum spanning tree enumeration problem, one of the well-known problems in combinatorial optimization.

\section{GENERATING ALL INDEPENDENT SETS}

Let $p_{1}, p_{2}, \ldots, p_{N}$ be $N$ distinct particle yields. Assume $S$ to be an independent set containing the ratios of these particle yields denoted as $r_{1}, r_{2}, \ldots, r_{N-1}$. We associate an undirected graph $G$ to $S$ whose vertices are labeled by $p_{1}, p_{2}, \ldots, p_{N}$ and there is an edge $\overline{p_{i} p_{j}}$ between the vertices $p_{i}, p_{j}$, if either $p_{i} / p_{j}$ or $p_{j} / p_{i}$ is in $S$. Observe that if $\overline{p_{i} p_{j}}, \overline{p_{j} p_{k}}$ are edges of $G$ then the vertices $p_{i}$ and $p_{k}$ are connected through the vertex 
$p_{j}$. More generally, a path between two vertices $p_{i}$ and $p_{j}$ is said to exist if either there is an edge between them or they can be connected through some or all of the remaining $N-2$ vertices. Since each of the particle yields $p_{1}, p_{2}, \ldots, p_{N}$ must appear at least in one of the ratios in $S$, then every vertex of $G$ is connected to at least one of the remaining $N-1$ vertices. Independence of $S$ also implies that for each $1 \leqslant k \leqslant N-1$

$$
r_{k} \neq \prod_{l=1}^{N-1} r_{l}^{s_{l}}, \quad \text { where } s_{l}=0, \pm 1 .
$$

The above equation imposes the condition that if $\overline{p_{i} p_{j}}$ is an edge of $G$ then there is no other path between $p_{i}$ and $p_{j}$. Equivalently, between any two vertices of $G$ there is a unique path between them. Hence, $G$ is a tree on the $N$ vertices labeled by $p_{1}, p_{2}, \ldots, p_{N}$. Conversely, every tree on $N$ vertices $p_{1}, p_{2}, \ldots, p_{N}$ gives rise to an independent set. Therefore, we have a one-to-one correspondence between the trees on $N$ vertices labeled by $p_{1}, p_{2}, \ldots, p_{N}$ and the independent sets.

For example, let us consider four distinct particles $p_{1}, p_{2}, p_{3}, p_{4}$. Then we have the following 16 independent sets:

$$
\begin{aligned}
I_{1}=\left\{\frac{p_{2}}{p_{1}}, \frac{p_{3}}{p_{1}}, \frac{p_{4}}{p_{1}}\right\}, & I_{2}=\left\{\frac{p_{2}}{p_{1}}, \frac{p_{4}}{p_{1}}, \frac{p_{3}}{p_{2}}\right\}, \\
I_{3}=\left\{\frac{p_{2}}{p_{1}}, \frac{p_{3}}{p_{1}}, \frac{p_{4}}{p_{3}}\right\}, & I_{4}=\left\{\frac{p_{2}}{p_{1}}, \frac{p_{3}}{p_{1}}, \frac{p_{4}}{p_{2}}\right\}, \\
I_{5}=\left\{\frac{p_{2}}{p_{1}}, \frac{p_{3}}{p_{2}}, \frac{p_{4}}{p_{2}}\right\}, & I_{6}=\left\{\frac{p_{2}}{p_{1}}, \frac{p_{4}}{p_{1}}, \frac{p_{4}}{p_{3}}\right\}, \\
I_{7}=\left\{\frac{p_{3}}{p_{1}}, \frac{p_{4}}{p_{1}}, \frac{p_{3}}{p_{2}}\right\}, & I_{8}=\left\{\frac{p_{3}}{p_{1}}, \frac{p_{3}}{p_{2}}, \frac{p_{4}}{p_{2}}\right\}, \\
\left.I_{11}=\left\{\frac{p_{3}}{p_{1}}, \frac{p_{3}}{p_{2}}, \frac{p_{4}}{p_{3}}\right\}, \frac{p_{4}}{p_{2}}, \frac{p_{4}}{p_{3}}\right\}, & I_{10}=\left\{\frac{p_{2}}{p_{1}}, \frac{p_{3}}{p_{2}}, \frac{p_{4}}{p_{3}}\right\}, \\
I_{13}=\left\{\frac{p_{4}}{p_{1}}, \frac{p_{4}}{p_{2}}, \frac{p_{4}}{p_{3}}\right\}, & \left.I_{14}=\left\{\frac{p_{3}}{p_{1}}, \frac{p_{4}}{p_{1}}, \frac{p_{4}}{p_{2}}\right\}, \frac{p_{3}}{p_{2}}, \frac{p_{4}}{p_{3}}\right\}, \\
I_{15}=\left\{\frac{p_{4}}{p_{1}}, \frac{p_{3}}{p_{2}}, \frac{p_{4}}{p_{2}}\right\}, & I_{16}=\left\{\frac{p_{3}}{p_{1}}, \frac{p_{4}}{p_{2}}, \frac{p_{4}}{p_{3}}\right\},
\end{aligned}
$$

These sets can be obtained by associating a unique tree $G_{m}$ to every independent set $I_{m}$ for all $1 \leqslant m \leqslant 16$ as shown in Fig. 1. Here the vertices symbolize particle yields. Each diagram in Fig. 1 corresponds to one set of independent ratios, where the ratios are represented by lines joining two vertices. There are six distinct ratios (unique up to inverse) for $N=4$, which are denoted by different colors. In general, the total number of different trees on $N$ labeled vertices is equal to $N^{N-2}$, which is given by Cayley's formula [58,59]. In fact the generation of those $N^{N-2}$ many trees is equivalent to generating all spanning trees of the complete graph on $N$ vertices labeled by $p_{1}, p_{2}, \ldots, p_{N}$. This is a particular case of the minimum spanning tree enumeration problem. This problem is important in its own right due to its wide range of applications, including telecommunication networks, and
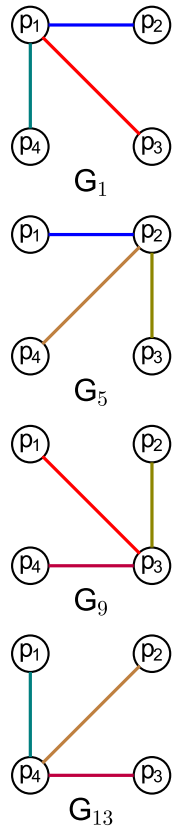

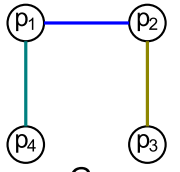

$\mathrm{G}_{2}$
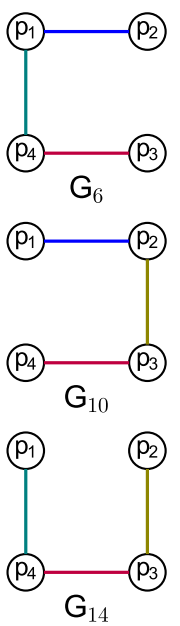

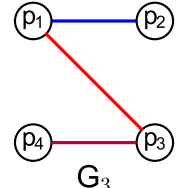

$\mathrm{G}_{3}$

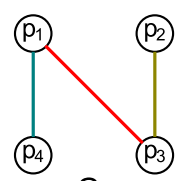

$\mathrm{G}_{7}$
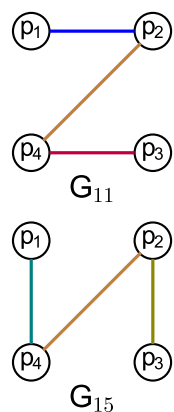

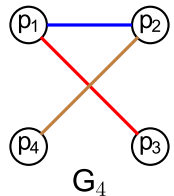

$\mathrm{G}_{4}$

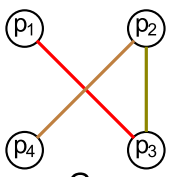

$\mathrm{G}_{8}$

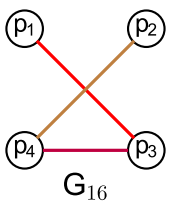

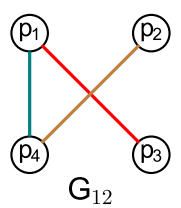

FIG. 1. All possible tree diagrams for the $N=4$ case. The vertices symbolize particle yields. Each diagram corresponds to one set of independent ratios, where the ratios are represented by lines joining two vertices. There are six distinct ratios (unique up to inverse) which are denoted by different colors.

therefore several algorithms with improvised efficiencies have been developed since mid 1950s. We refer to [60-62] and the references therein for more details.

The algorithm we have employed to generate all trees on $N$ labeled vertices is as follows. It is straightforward to check that there can be $\frac{N(N-1)}{2}$ different edges connecting any two vertices $p_{i}, p_{j}$ considering $1 \leqslant i, j \leqslant N$ and $i \neq j$. By implementing the algorithm, at every step we choose $N-1$ edges, say $e_{1}, e_{2}, \ldots, e_{N-1}$ (without repetition), from $\frac{N(N-1)}{2}$ edges and denote the graph formed with $N$ vertices labeled by $p_{1}, p_{2}, \ldots, p_{N}$ and edges $e_{1}, e_{2}, \ldots, e_{N-1}$ by $G^{\prime}$. In the next step, we shall verify whether the vertex $p_{i}$, for all $1 \leqslant i \leqslant N$, is connected to any other vertex by an edge or not. Then we construct the incidence matrix $M=\left(m_{i j}\right)_{N \times(N-1)}$ to verify whether the graph $G^{\prime}$ is a tree. The entries of $M$ are defined as follows: $m_{i j}=1$ whenever the edge $e_{j}$ joins the vertex $p_{i}$ to some other vertex, and 0 otherwise, for all $1 \leqslant i, j \leqslant N$. Observe that, if we delete one of the rows of $M$ then we obtain a submatrix of $M$ of size $(N-1) \times(N-1)$. We continue this process for each of the $N$ rows of $M$ and obtain $N$ many submatrices of $M$. Then we check whether the rank of each of those $N$ submatrices of $M$ is $N-1$. If it is true then we conclude that $G^{\prime}$ is a tree. In this way, we generate all $N^{N-2}$ trees on $N$ vertices and, consequently, we obtain all $N^{N-2}$ possible independent sets of ratios for our analysis.

\section{DATA ANALYSIS}

We have used RHIC [63-78] and LHC [79-82] data at midrapidity for the most central collisions for our analysis. All hadrons with masses up to $2 \mathrm{GeV}$, with known degrees of 
freedom, are included for the HRG spectrum. The masses and branching ratios used are as given in Refs. [52,83]. It is to be noted that we have used vacuum masses for all the hadrons, as the in-medium mass modification in connection with chiral symmetry restoration does not have any significant effect on freeze-out parameters for higher collision energies [84-86]. We could not find the $\bar{\Lambda}$ yield at LHC. Therefore, at this energy, we assumed $\bar{\Lambda}$ yield to be same as that reported for $\Lambda$. Therefore, in order to obtain the freeze-out parameters, we have used the hadron yields of $\pi^{ \pm}(139.57 \mathrm{MeV}), k^{ \pm}$(493.68 $\mathrm{MeV}), p, \bar{p}(938.27 \mathrm{MeV}), \Lambda, \bar{\Lambda}(1115.68 \mathrm{MeV})$, and $\Xi^{\mp}$ (1321.71 MeV).

To obtain the freeze-out parameters it is a common approach to fix $\mu_{Q}$ and $\mu_{S}$ using the following constraint relations [45]:

$$
\frac{\sum_{i} n_{i}\left(T, \mu_{B}, \mu_{S}, \mu_{Q}\right) B_{i}}{\sum_{i} n_{i}\left(T, \mu_{B}, \mu_{S}, \mu_{Q}\right) Q_{i}}=\mathrm{constant}
$$

and

$$
\sum_{i} n_{i}\left(T, \mu_{B}, \mu_{S}, \mu_{Q}\right) S_{i}=0 .
$$

With these two constraint equations, the problem is reduced to a two-dimensional problem. The constant value of the ratio of net baryon number and net charge depends on the physical system. For example, in $\mathrm{Au}+\mathrm{Au}$ collisions, this constant is given by $\left(N_{p}+N_{n}\right) / N_{p}=2.5$, with $N_{p}$ and $N_{n}$ denoting the number of protons and neutrons in the colliding nuclei. However for LHC $\left(\sqrt{s_{\mathrm{NN}}}=2.76 \mathrm{TeV}\right)$ and RHIC $\left(\sqrt{s_{\mathrm{NN}}}=\right.$ $200 \mathrm{GeV}$ ) energies, baryon stopping is expected to be negligible. In such cases, net baryon number and net charge may be vanishingly small and therefore their ratio may become arbitrary in a rapidity bin. Thus, it seems natural to relax the constraint given in Eq. (7) to extract freeze-out parameters by $\chi^{2}$ minimization. In the present analysis, we consider both cases: we call Model-I the case where the constraint given in Eq. (7) is imposed and Model-II the case where we do not enforce this constraint relation to hold.

For $N=10$ observed hadron yields, we first obtained $10^{8}$ independent sets of nine ratios, as explained in Sec. III. We then performed $\chi^{2}$ minimization fits for each set using our numerical code for both Model-I and Model-II with a convergence criterion of $10^{-4}$ or better. This procedure to fit all possible independent sets results in $10^{8}$ values of each extracted freeze-out parameter and corresponding $\chi^{2}$ numbers. We then construct histograms for each extracted freeze-out parameter, as well as for the $\chi^{2}$ values. However, histograms give an overall notion about the density of the underlying distribution of the data. Therefore, for further analysis, we perform a least-square fit to histograms of each parameter with Gaussian distribution. The fitted distribution curves quantify the probability distribution function for a population that has the maximum likelihood of producing the distribution that exists in the given sample. The mean of the Gaussian distribution corresponding to a given freeze-out parameter leads to an estimate of the central value of that parameter. The systematic uncertainties arising from the fitting procedure is calculated from the full width at half maximum $(2.3548 \sigma)$, where $\sigma$ is the standard deviation of the Gaussian distribution.

\section{RESULTS AND DISCUSSIONS}

In this section, we discuss the extracted chemical freezeout parameters for center-of-mass collision energies $\sqrt{s_{\mathrm{NN}}}=$ $200 \mathrm{GeV}$ at RHIC and $\sqrt{s_{\mathrm{NN}}}=2.76 \mathrm{TeV}$ at LHC. We fit $10^{8}$ sets of yield ratios to obtain a distribution for fit parameters, i.e., freeze-out temperatures $T$ and freeze-out chemical potentials $\mu_{B}, \mu_{Q}$, and $\mu_{S}$. The estimated values of the parameters are derived from the mean of the fitted Gaussian distribution. The error bars quantify the variations of the parameters obtained from all possible sets of independent ratios. Results including the constraint, Eq. (7), on net baryon and net charge (Model-I) and those for the unconstrained system (Model-II) are shown separately. Finally we also compare our results with those obtained earlier in literature.

Histogram distribution and corresponding Gaussian fit to this distribution for freeze-out temperatures are shown for center of mass energy $\sqrt{s_{\mathrm{NN}}}=200 \mathrm{GeV}$ in Fig. 2(a) and $\sqrt{s_{\mathrm{NN}}}=2.76 \mathrm{TeV}$ in Fig. 2(b). The qualitative as well as the quantitative estimations, for both Model-I and Model-II, are consistent with each other. We find that for RHIC the extracted mean value of freeze-out temperature is $169 \mathrm{MeV}$. which is slightly larger than the earlier estimates. On the other hand, for LHC, we find the fitted mean value to be 155 $\mathrm{MeV}$. Nevertheless the estimated values lies near the values reported in literature, where the analysis was done with a particular set of independent ratios as documented in Tables I and in II. Our analysis shows that the present model has a small systematic uncertainty in chemical freeze-out temperature, which is quite promising. In general, the freeze-out temperature increases with increasing $\sqrt{s_{\mathrm{NN}}}$ and approaches a saturation [87], except at the LHC energy. As expected, in our analysis also, the temperature is lower at LHC energy than at RHIC energy, due to the lower yield of protons at LHC [82].

In Fig. 3, we show the histogram distribution and the Gaussian fit to this distribution for baryon chemical potential $\mu_{B}$ extracted from all independent sets. In Fig. 3(a) we present our results for $\sqrt{s_{\mathrm{NN}}}=200 \mathrm{GeV}$ collision energy whereas in Fig. 3(b) results for $\sqrt{s_{\mathrm{NN}}}=2.76 \mathrm{TeV}$ are shown. Generally, for lower collision energies, a significant number of baryons deposit their energies in the vicinity of the center-of-mass frame due to baryon stopping. But as the energy increases colliding baryons may become almost transparent to each other and deposit their energy outside the collision region. In our analysis for both the models, we have estimated the value of $\mu_{B}$ and quantified the systematic uncertainty for high collision energies. The comparison with several literature results shows our derived values lie in the range of their predicted values; see Tables I and II for reference.

Due to the possible redistribution of Fermi momentum among larger degrees of freedom, strangeness production at higher baryon densities is conjectured to be dominant. The nonzero value of baryon chemical potential induces the production of strange baryons, which imminently requires the strange chemical potential to produce enough strange antiparticles. The existence of this nonzero strange chemical potential $\mu_{S}$ ensures the vanishing of the net strangeness condition of the colliding nuclei. The appearance of nonzero $\mu_{S}$ 


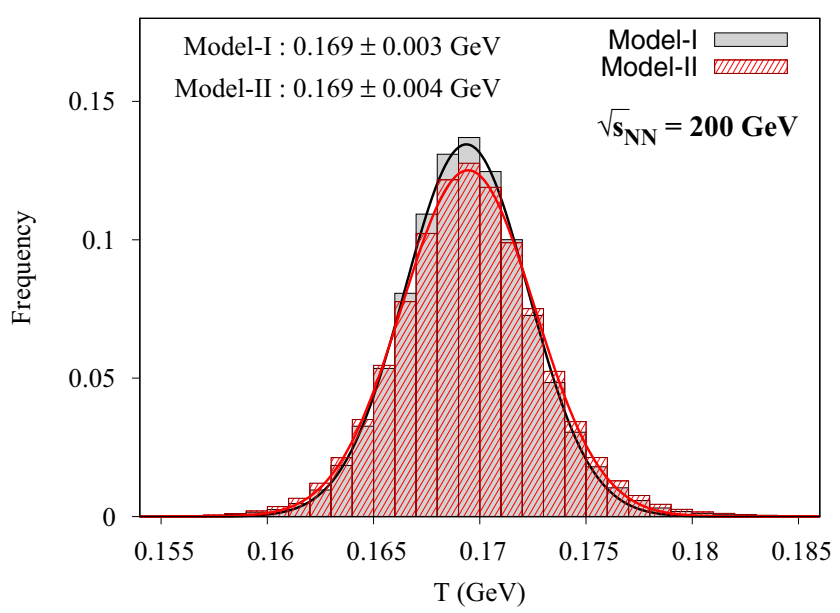

(a)

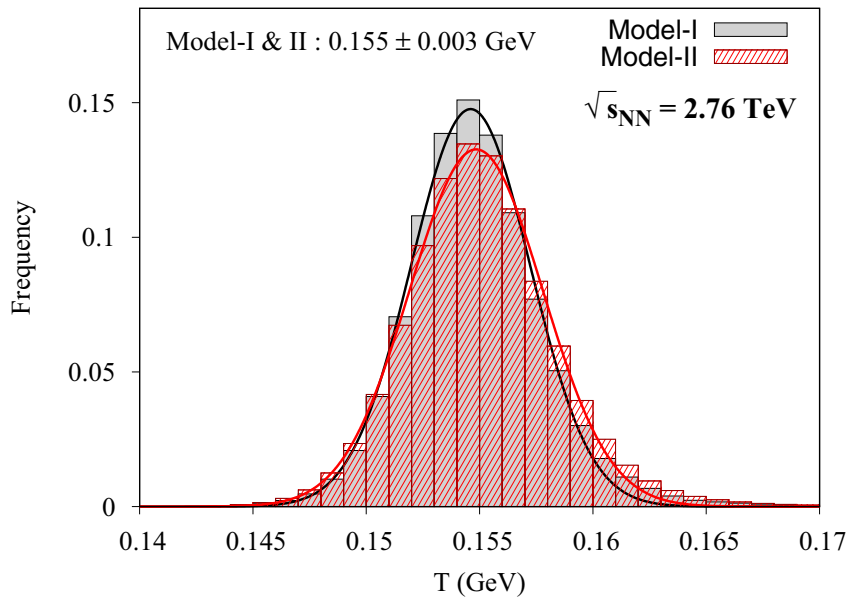

(b)

FIG. 2. The distribution of chemical freeze-out temperatures for collision energies $\sqrt{s_{\mathrm{NN}}}=200 \mathrm{GeV}$ (RHIC) and $\sqrt{s_{\mathrm{NN}}}=2.76 \mathrm{TeV}$ (LHC). Also shown are the fitted Gaussian curves, and the values of extracted freeze-out temperature with systematic error bars are listed, for both Model-I and Model-II.

TABLE I. Comparison of the values of freeze-out parameters with recent literature for $\sqrt{s_{\mathrm{NN}}}=200 \mathrm{GeV}$ at RHIC.

\begin{tabular}{|c|c|c|c|c|c|c|}
\hline $\begin{array}{l}\text { Parameters } \\
\text { for RHIC }\end{array}$ & Model-I & Model-II & Andronic et al. [21] & STAR BES [55] & \multicolumn{2}{|c|}{ Bhattacharyya et al. [56] } \\
\hline$T(\mathrm{GeV})$ & $0.169 \pm 0.003$ & $0.169 \pm 0.004$ & $0.160 \pm 0.006$ & $0.164 \pm 0.005$ & $0.165_{-0.010}^{+0.008}$ & $0.166_{-0.012}^{+0.010}$ \\
\hline$\mu_{B}(\mathrm{GeV})$ & $0.028 \pm 0.010$ & $0.030 \pm 0.016$ & $0.020 \pm 0.004$ & $0.028 \pm 0.006$ & $0.028_{-0.015}^{+0.010}$ & $0.032_{-0.017}^{+0.012}$ \\
\hline$\mu_{S}(\mathrm{GeV})$ & $0.007 \pm 0.003$ & $0.009 \pm 0.010$ & & $0.006 \pm 0.004$ & $0.007_{-0.003}^{+0.015}$ & $0.009_{-0.008}^{+0.006}$ \\
\hline$\mu_{Q}(\mathrm{GeV})$ & $-0.001 \pm 0.0004$ & $-0.004 \pm 0.018$ & & & $-0.0008_{-0.0003}^{+0.00004}$ & $-0.005_{-0.004}^{+0.008}$ \\
\hline
\end{tabular}

TABLE II. Comparison of the values of freeze-out parameters with recent literature for $\sqrt{s_{\mathrm{NN}}}=2.76 \mathrm{TeV}$ at LHC.

\begin{tabular}{lccrr}
\hline \hline \multirow{2}{*}{$\begin{array}{l}\text { Parameters } \\
\text { for LHC }\end{array}$} & \multicolumn{1}{c}{ Model-I } & Model-II & Andronic et al. [40] & Bhattacharyya et al. [56] \\
\hline$T(\mathrm{GeV})$ & $0.155 \pm 0.003$ & $0.155 \pm 0.003$ & $0.157 \pm 0.002$ & WC \\
$\mu_{B}(\mathrm{GeV})$ & $0.002 \pm 0.009$ & $0.002 \pm 0.015$ & $0.007 \pm 0.004$ & $0.152_{-0.006}^{+0.008}$ \\
$\mu_{S}(\mathrm{GeV})$ & $0.0006 \pm 0.0018$ & $0.0005 \pm 0.0089$ & & $0.003_{-0.013}^{+0.013}$ \\
$\mu_{Q}(\mathrm{GeV})$ & $-0.00005 \pm 0.0002$ & $-0.0004 \pm 0.016$ & & $0.0005_{-0.0028}^{+0.0023}$ \\
\hline \hline
\end{tabular}

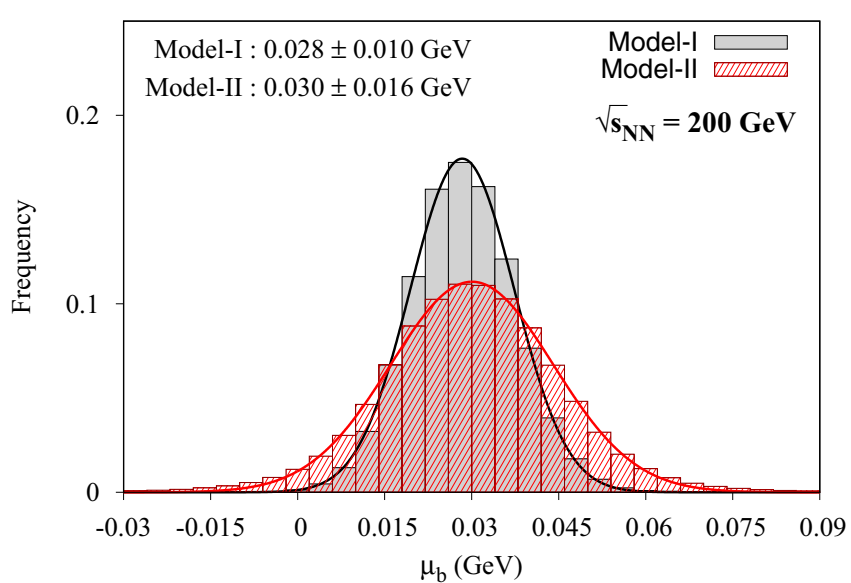

(a)

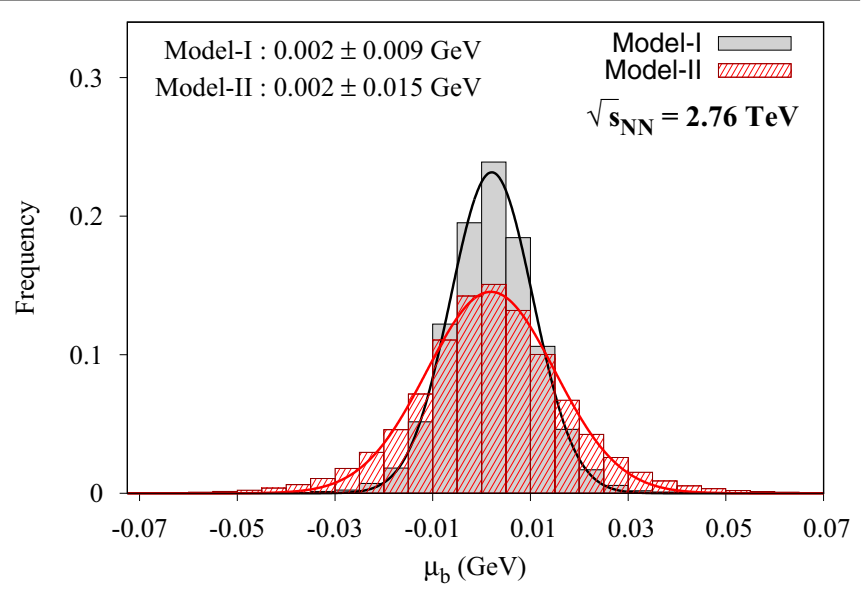

(b)

FIG. 3. The distribution of baryon chemical potential at chemical freeze-out for collision energies $\sqrt{s_{\mathrm{NN}}}=200 \mathrm{GeV}(\mathrm{RHIC})$ and $\sqrt{s_{\mathrm{NN}}}=$ $2.76 \mathrm{TeV}$ (LHC). Also shown are the fitted Gaussian curves, and the values of extracted baryon chemical potential freeze-out with systematic error bars are listed, for both Model-I and Model-II. 


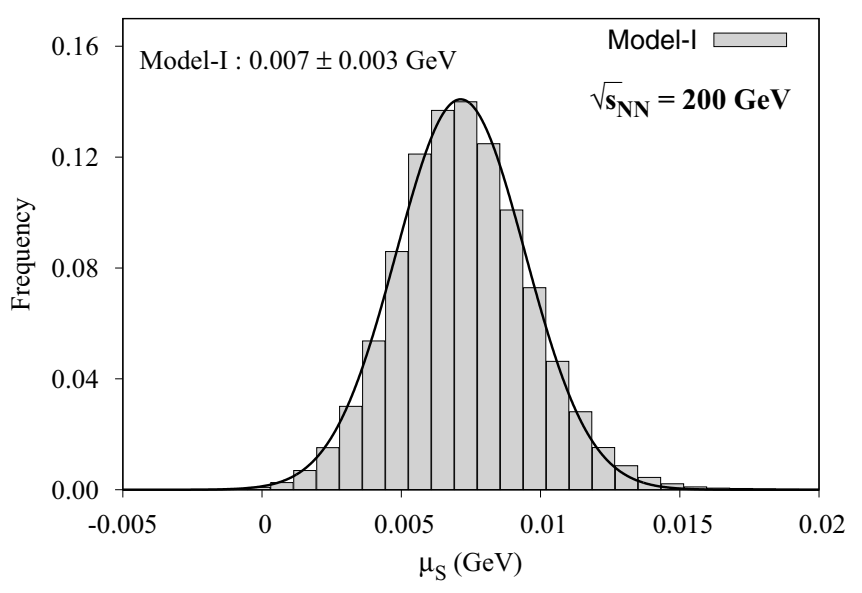

(a)

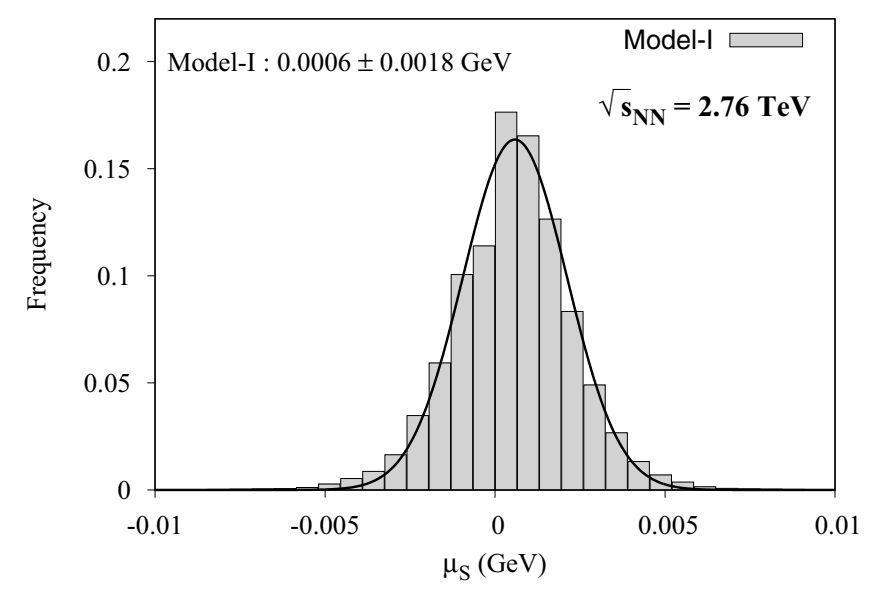

(c)

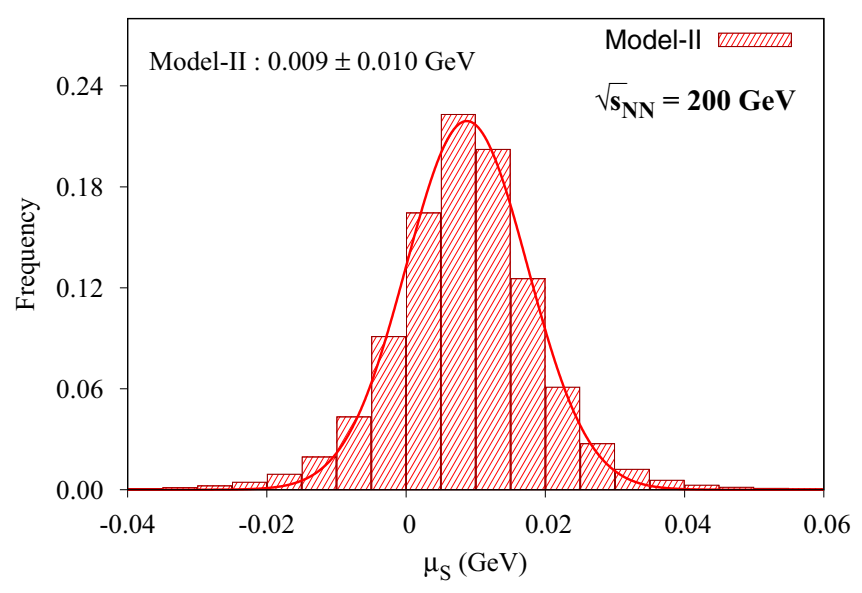

(b)

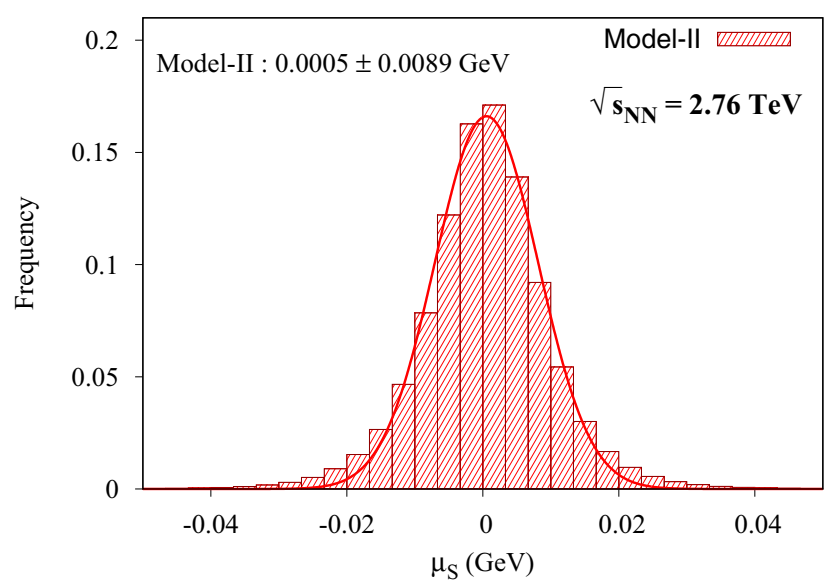

(d)

FIG. 4. The distribution of strange chemical potential at chemical freeze-out for collision energies $\sqrt{s_{\mathrm{NN}}}=200 \mathrm{GeV}(\mathrm{RHIC})$ and $\sqrt{s_{\mathrm{NN}}}=$ $2.76 \mathrm{TeV}$ (LHC). Also shown are the fitted Gaussian curves, and the values of extracted strange chemical potential at freeze-out with systematic error bars are listed, for both Model-I and Model-II.

is considered to be an indication of chemical equilibration in strongly interacting matter [88]. As the collision energy increases, $\mu_{S}$ eventually decreases. On the other hand, electric charge chemical potential $\mu_{Q}$ appears due to the net isospin of the colliding nuclei. While scanning the collision energy range, $\mu_{Q}$ remains nonzero but quite small and eventually approaches to zero at higher $\sqrt{s_{\mathrm{NN}}}$. Several analyses thus approximated $\mu_{Q}$ to be zero at higher collision energies [55]. In our analysis, we have estimated the values of $\mu_{S}$ and $\mu_{Q}$ using both Model-I and Model-II.

The mean values of $\mu_{S}$ are in reasonable agreement with each other for both the models, as illustrated in Figs. 4(a) and 4(b) for RHIC energy. Though the estimated values are commensurate for both models, Model-II has much larger systematic uncertainty, and exclusion of the constraint is the source of this uncertainty. The same features are also observed for LHC energy as shown in Figs. 4(c) and 4(d).

Furthermore, as shown in Figs. 5(a) and 5(b), the numerical value of $\mu_{Q}$ is small. Especially for LHC energy, as shown in Figs. 5(c) and 5(d), it is vanishingly small. Therefore, exclusion of the net baryon to net charge constraint in ModelII results in fluctuations. Only the $\chi^{2}$ analysis using these parameters can confirm the reliability of Model-II, which will be discussed later.

For comparison, the freeze-out parameters from relevant literature are also documented in Tables I and II for collision energies of $\sqrt{s_{\mathrm{NN}}}=200 \mathrm{GeV}$ at RHIC and $\sqrt{s_{\mathrm{NN}}}=2.76 \mathrm{TeV}$ at LHC, respectively. Specifically we have used results from Refs. [21,55,56] for RHIC energy and Refs. [40,56] for LHC energy. For RHIC, we obtain a slightly larger value of the extracted freeze-out temperature. Nevertheless, we find that our extracted values of freeze-out parameters are more or less in agreement with those reported earlier in literature. However, one must keep in mind that there are significant differences in the analysis procedure and computation techniques employed here. The error bars considered in Ref. [21] are obtained by varying the $\chi^{2}$ by unity, which accounts for the fitting error. In Ref. [55], $\mu_{Q}$ was assumed to be vanishingly small and was therefore neglected. However, an additional parameter $\gamma_{s}$ was introduced to account for the possible deviation of 


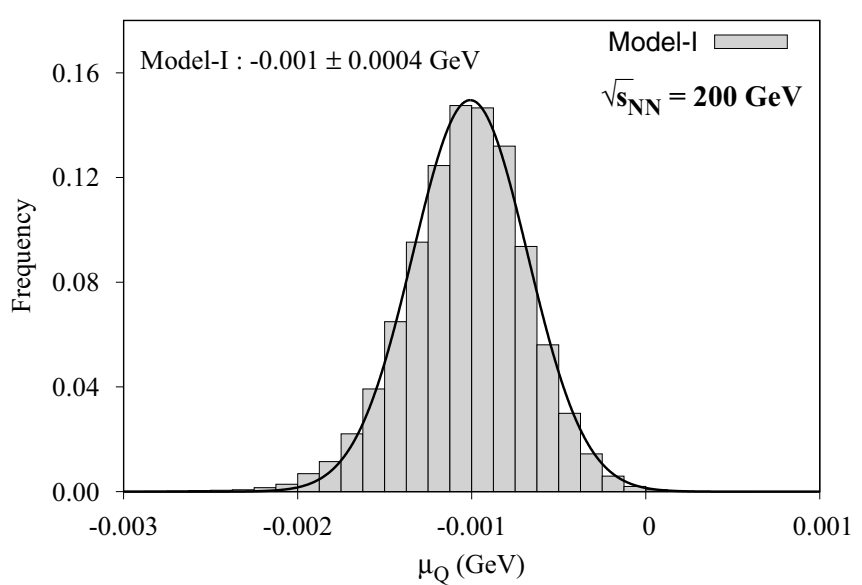

(a)

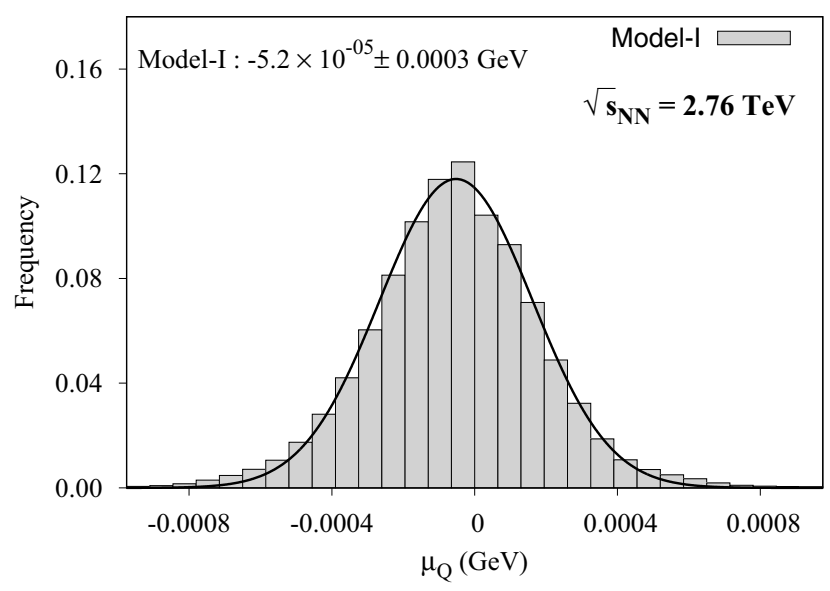

(c)

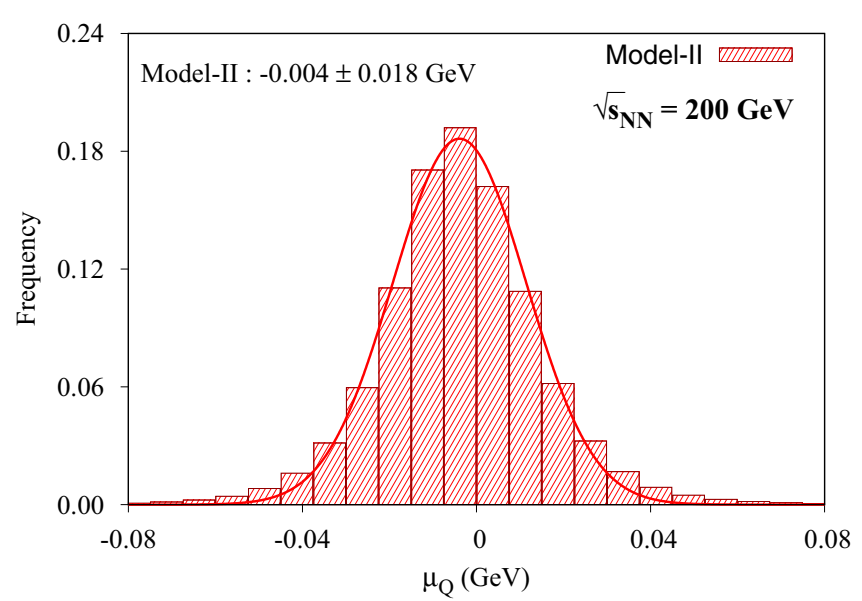

(b)

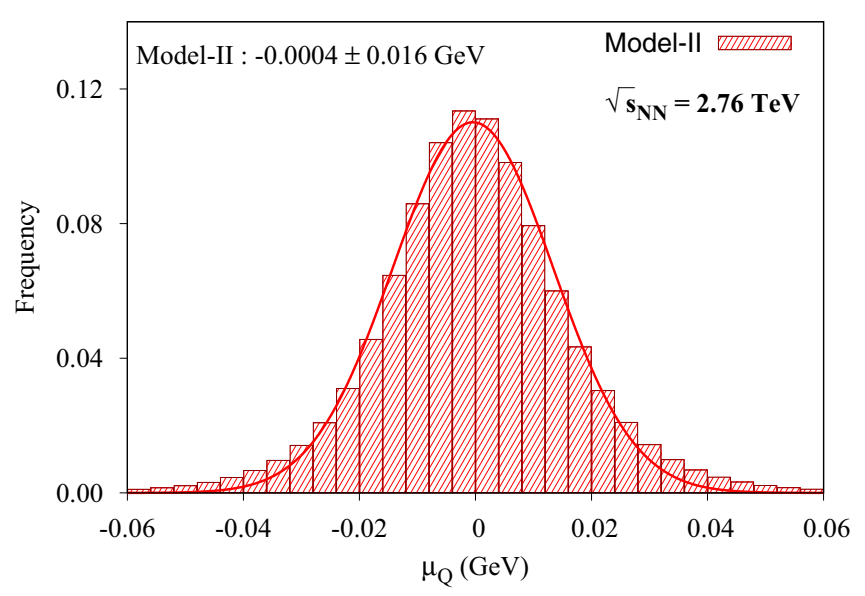

(d)

FIG. 5. The distribution of electric charge chemical potential at chemical freeze-out for collision energies $\sqrt{s_{\mathrm{NN}}}=200 \mathrm{GeV}$ (RHIC) and $\sqrt{s_{\mathrm{NN}}}=2.76 \mathrm{TeV}$ (LHC). Also shown are the fitted Gaussian curves, and the values of extracted electric charge chemical potential at freeze-out with systematic error bars are listed, for both Model-I and Model-II.

strange particle abundances from chemical equilibrium. The only ratios that are used in the analysis of Ref. [55] are $\pi^{-} / \pi^{+}, k^{-} / k+, \bar{p} / p, \bar{\Lambda} / \Lambda, \Xi^{+} / \Xi^{-}, k^{-} / \pi^{-}, \bar{p} / \pi^{-}, \Lambda / \pi^{-}$, and $\Xi^{+} / \pi^{-}$. They also studied different sources of systematic uncertainties, which propagates from yield to freeze-out parameters. In Ref. [56] the systematic error bars were calculated with nine independent sets, considering the usual constraint of strong charges (WC) and without constrain relations (WOC) of Eqs. (7) and (8). In Ref. [40], the authors considered system volume as a parameter and the standard deviations quoted there come only from experimental uncertainties.

Reduced $\chi^{2}$, i.e., $\chi^{2} / \mathrm{NDF}$, gives a measure of goodness of fit, where NDF stands for the number of degrees of freedom. The usual practice is to choose a specific independent set so that the $\chi^{2}$ is equal to the number of degrees of freedom of the system. The histogram distribution of reduced $\chi^{2}$ corresponding to all possible independent sets for $\sqrt{s_{\mathrm{NN}}}=2.76 \mathrm{TeV}$
(LHC) and $\sqrt{s_{\mathrm{NN}}}=200 \mathrm{GeV}$ (RHIC) are shown for both Model-I and Model-II in Fig. 6. We see that $\chi^{2} / \mathrm{NDF}$ for the models differ only slightly. It is interesting to see that the distribution is peaked at $\chi^{2} / \mathrm{NDF} \simeq 1$ for RHIC, as shown in Fig. 6(a), indicating a good fit. On the other hand, from Fig. 6(b), we see that $\chi^{2} / \mathrm{NDF} \simeq 2.2$ for LHC. This suggests that quality of fit at LHC may not be as good as that at RHIC. Anyhow, this result is not surprising as it has been pointed out in existing literature that reduced $\chi^{2}$ for LHC is quite large, if we consider the equilibration of all the hadrons simultaneously within the framework of the conserved charges $[46,89]$. However, the usual practice is to minimize $\chi^{2}$ for a specific independent set, and a large value of $\chi^{2} / \mathrm{NDF}$ for that particular set is not necessarily alarming. But, we have done the study for all possible independent sets and observe that the peak of the reduced $\chi^{2}$ distribution lies near 2.2, for both models. Thus, the thermal model analysis for LHC data demands further study. 


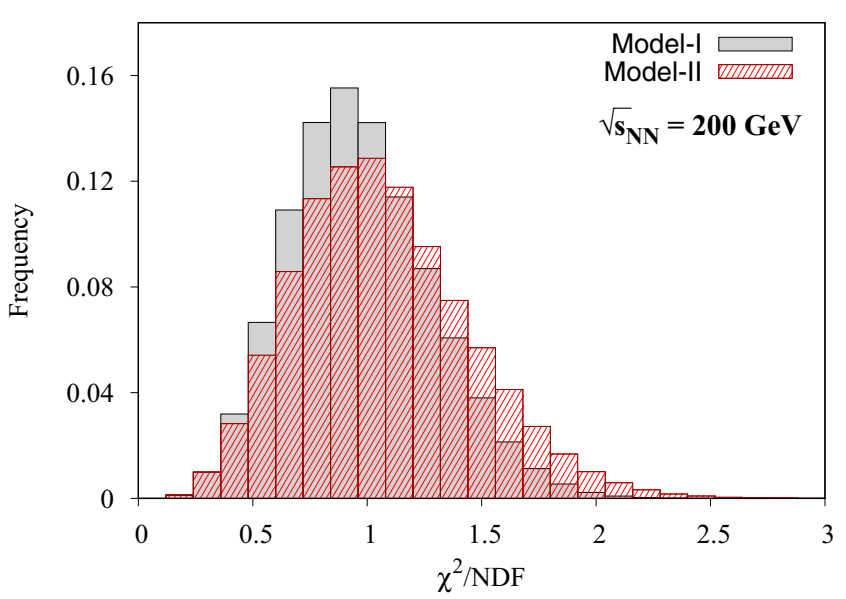

(a)

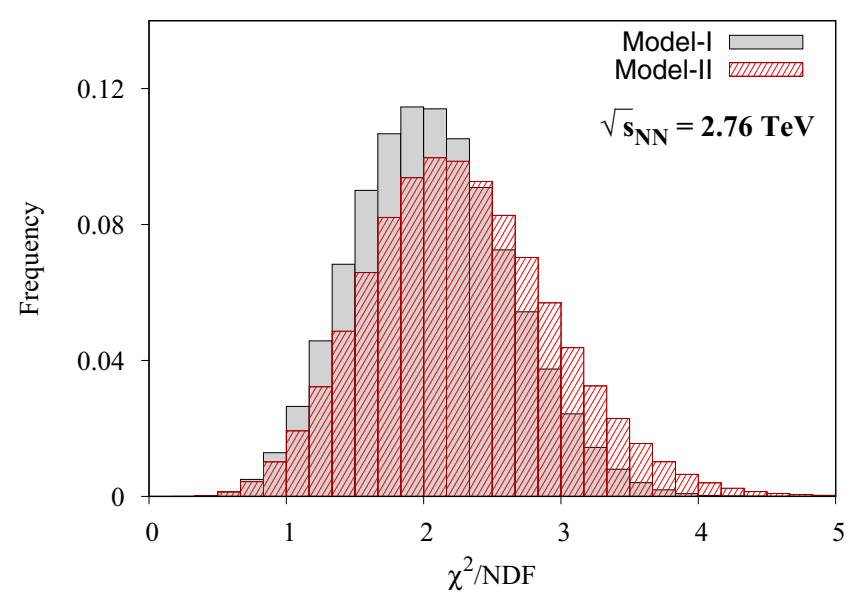

(b)

FIG. 6. The histogram distribution of $\chi^{2} / \mathrm{NDF}$ for all possible independent sets for collision energies $\sqrt{s_{\mathrm{NN}}}=200 \mathrm{GeV}$ (RHIC) and $\sqrt{s_{\mathrm{NN}}}=2.76 \mathrm{TeV}$ (LHC), for both Model-I and Model-II.

\section{SUMMARY AND OUTLOOK}

It has been conjectured in existing literature that $\chi^{2}$ fit of experimentally measured hadronic yields, or multiplicity ratios, within the thermal model framework can successfully extract chemical freeze-out parameters to a certain extent. It is evident that there are many possible sources of systematic uncertainties that can affect the analysis. The usual practice is to choose a specific set of independent ratios for the analysis, such that $\chi^{2} / \mathrm{NDF}$ is close to 1 . This is one of the sources of systematic uncertainty, as the equilibration parameters are biased to the chosen particle ratios. Estimation of these uncertainties is important because it would shed light on the reliability of such thermal models where we have assumed a grand canonical ensemble with thermodynamic equilibration of all the hadrons emerging from the heavy-ion collision experiments.

In this article, we have provided an elegant method to quantify such systematic uncertainties in the chemical freezeout parameters extracted from thermal model analysis of hadron multiplicity ratios. We have identified the enumeration problem of independent sets to a well known problem in combinatorial optimization and graph theory. By implementing the minimum spanning tree algorithm, we have generated all possible independent sets. This corresponds to the number of all different trees on $N$ vertices resulting in generation of $N^{N-2}$ independent sets as expected from Cayley's formula.

We have performed the $\chi^{2}$ minimization on each set to obtain freeze-out parameters corresponding to yield ratios of experimental data at collision energies of $\sqrt{s_{\mathrm{NN}}}=200 \mathrm{GeV}$ at RHIC and $\sqrt{s_{\mathrm{NN}}}=2.76 \mathrm{TeV}$ at LHC. Since the number of sets is extremely large $\left(10^{8}\right)$, we obtain a distribution of these parameters. From these distributions, we estimate the mean value of the said parameter along with the quantitative measure of systematic uncertainty. Model-II seems to have larger systematic uncertainty than Model-I, which is expected because Model-II has one extra free parameter. But the $\chi^{2} / \mathrm{NDF}$ distribution for RHIC is peaked around 1, which ensures that the systematics are under control for both the models. One may be tempted to conclude that a global equilibration scenario is achieved for RHIC where equilibration of all hadrons occurs simultaneously. On the other hand, for LHC we found that, although the parameters seem to be in good agreement with existing literature, the value of the $\chi^{2} / \mathrm{NDF}$ distribution is peaked is away from 1 . This leads to a serious challenge about the applicability of the thermal model (in the present form) at LHC energy.

At this juncture, we would like to emphasize that there are no empirical reasons to believe that the simplistic statistical hadronization models can explain the entire physical scenario near the crossover region [90,91]. The uncertainties in the prediction at LHC energy leads to study of alternative freezeout schemes, such as [92-94]. Moreover, even within thermal model analysis, there is a possibility of a mass dependent or flavor dependent sequential freeze-out scenario. The intrinsic limitation of these statistical models is that one does not have precise knowledge of how different ratios are dependent on the physical scenario. On the other hand, our method of estimating systematic uncertainties by generating all possible sets of independent ratios is quite general and is applicable also for data sets with bias. For instance, if one could estimate the bias on the ratios then the present method could be employed to fit all possible sets and calculate the weighted average of the fitted parameters and corresponding standard deviations. In the absence of that knowledge, the present analysis is the best one can do to extract systematic errors, which we have estimated in the present article.

Looking forward, the framework presented here can be applied to other collision energies at RHIC and LHC in order to discern whether the extraction of chemical freeze-out parameters is biased by the choice of yield ratios. Moreover, the current framework can be easily extended to physics analysis using double or multiple ratios, which will have important implications for sequential freeze-out models. We leave these for future work. 


\section{ACKNOWLEDGMENTS}

S.B. thanks Deeptak Biswas and Rajarshi Ray for helpful discussions and acknowledges NISER for kind hospitality. A.J. thanks Tuhin Ghosh and Kush Saha for their help in using the VIRGO computing cluster at NISER. S.R. thanks Rishiraj Bhattacharyya and Himadri Nayak for fruitful discussions. S.B. was supported by CSIR and DST. A.J. and S.R. are supported in part by the DST-INSPIRE faculty award under Grants No. DST/INSPIRE/04/2017/000038 and No. DST/INSPIRE/04/2016/000215, respectively.
[1] E. Fermi, Prog. Theor. Phys. 5, 570 (1950).

[2] I. Ya. Pomeranchuk, Dokl. Akad. Nauk Ser. Fiz. 78, 889 (1951).

[3] L. D. Landau, Izv. Akad. Nauk Ser. Fiz. 17, 51 (1953).

[4] S. Z. Belenkij and L. D. Landau, Usp. Fiz. Nauk 56, 309 (1955) [Nuovo Cim. Suppl. 3(S10), 15 (1956)].

[5] R. Hagedorn and J. Rafelski, Phys. Lett. 97B, 136 (1980).

[6] D. H. Rischke, M. I. Gorenstein, H. Stoecker, and W. Greiner, Z. Phys. C 51, 485 (1991).

[7] J. Cleymans, M. I. Gorenstein, J. Stalnacke, and E. Suhonen, Phys. Sc 48, 277 (1993).

[8] P. Braun-Munzinger, J. Stachel, J. P. Wessels, and N. Xu, Phys. Lett. B 344, 43 (1995).

[9] J. Cleymans, D. Elliott, H. Satz, and R. L. Thews, Z. Phys. C 74, 319 (1997).

[10] G. D. Yen, M. I. Gorenstein, W. Greiner, and S.-N. Yang, Phys. Rev. C 56, 2210 (1997).

[11] U. W. Heinz, J. Phys. G 25, 263 (1999).

[12] J. Cleymans and K. Redlich, Phys. Rev. Lett. 81, 5284 (1998).

[13] P. Braun-Munzinger, I. Heppe, and J. Stachel, Phys. Lett. B 465, 15 (1999).

[14] J. Cleymans and K. Redlich, Phys. Rev. C 60, 054908 (1999).

[15] P. Braun-Munzinger, D. Magestro, K. Redlich, and J. Stachel, Phys. Lett. B 518, 41 (2001).

[16] F. Becattini, M. Gazdzicki, A. Keranen, J. Manninen, and R. Stock, Phys. Rev. C 69, 024905 (2004).

[17] P. Braun-Munzinger, K. Redlich, and J. Stachel, Quark-Gluon Plasma 3, 491 (2004).

[18] F. Karsch, K. Redlich, and A. Tawfik, Phys. Lett. B 571, 67 (2003).

[19] A. Tawfik, Phys. Rev. D 71, 054502 (2005).

[20] F. Becattini, J. Manninen, and M. Gazdzicki, Phys. Rev. C 73, 044905 (2006).

[21] A. Andronic, P. Braun-Munzinger, and J. Stachel, Nucl. Phys. A 772, 167 (2006).

[22] A. Andronic, P. Braun-Munzinger, and J. Stachel, Phys. Lett. B 673, 142 (2009); 678, 516(E) (2009).

[23] J. Manninen and F. Becattini, Phys. Rev. C 78, 054901 (2008).

[24] A. Andronic, P. Braun-Munzinger, J. Stachel, and M. Winn, Phys. Lett. B 718, 80 (2012).

[25] S. K. Tiwari, P. K. Srivastava, and C. P. Singh, Phys. Rev. C 85, 014908 (2012).

[26] V. V. Begun, M. Gazdzicki, and M. I. Gorenstein, Phys. Rev. C 88, 024902 (2013).

[27] J. Fu, Phys. Lett. B 722, 144 (2013).

[28] A. Tawfik, Phys. Rev. C 88, 035203 (2013).

[29] P. Garg, D. K. Mishra, P. K. Netrakanti, B. Mohanty, A. K. Mohanty, B. K. Singh, and N. Xu, Phys. Lett. B 726, 691 (2013).

[30] A. Bhattacharyya, S. Das, S. K. Ghosh, R. Ray, and S. Samanta, Phys. Rev. C 90, 034909 (2014).
[31] M. Albright, J. Kapusta, and C. Young, Phys. Rev. C 90, 024915 (2014).

[32] G. P. Kadam and H. Mishra, Phys. Rev. C 92, 035203 (2015).

[33] G. P. Kadam and H. Mishra, Phys. Rev. C 93, 025205 (2016).

[34] G. P. Kadam, arXiv:1510.04371.

[35] M. Albright, J. Kapusta, and C. Young, Phys. Rev. C 92, 044904 (2015).

[36] A. Bhattacharyya, R. Ray, S. Samanta, and S. Sur, Phys. Rev. C 91, 041901(R) (2015).

[37] A. Bhattacharyya, S. K. Ghosh, R. Ray, and S. Samanta, EPL 115, 62003 (2016).

[38] V. Begun, Phys. Rev. C 94, 054904 (2016).

[39] A. Bhattacharyya, S. K. Ghosh, S. Maity, S. Raha, R. Ray, K. Saha, S. Samanta, and S. Upadhaya, Phys. Rev. C 99, 045207 (2019).

[40] A. Andronic, P. Braun-Munzinger, K. Redlich, and J. Stachel, Nature (London) 561, 321 (2018).

[41] S. Ghosh, S. Ghosh, and S. Bhattacharyya, Phys. Rev. C 98, 045202 (2018).

[42] A. Dash, S. Samanta, and B. Mohanty, Phys. Rev. C 97, 055208 (2018).

[43] D. Biswas, arXiv:2003.10425.

[44] D. Biswas, Phys. Rev. C 102, 054902 (2020).

[45] P. Alba, W. Alberico, R. Bellwied, M. Bluhm, V. Mantovani Sarti, M. Nahrgang, and C. Ratti, Phys. Lett. B 738, 305 (2014).

[46] S. Chatterjee, S. Das, L. Kumar, D. Mishra, B. Mohanty, R. Sahoo, and N. Sharma, Adv. High Energy Phys. 2015, 349013 (2015).

[47] S. Chatterjee, D. Mishra, B. Mohanty, and S. Samanta, Phys. Rev. C 96, 054907 (2017).

[48] R. P. Adak, S. Das, S. K. Ghosh, R. Ray, and S. Samanta, Phys. Rev. C 96, 014902 (2017).

[49] J. Cleymans, H. Oeschler, K. Redlich, and S. Wheaton, Phys. Lett. B 615, 50 (2005).

[50] J. Cleymans, H. Oeschler, K. Redlich, and S. Wheaton, Phys. Rev. C 73, 034905 (2006).

[51] S. Chatterjee, R. M. Godbole, and S. Gupta, Phys. Lett. B 727, 554 (2013).

[52] S. Wheaton and J. Cleymans, Comput. Phys. Commun. 180, 84 (2009).

[53] G. Torrieri, S. Steinke, W. Broniowski, W. Florkowski, J. Letessier, and J. Rafelski, Comput. Phys. Commun. 167, 229 (2005).

[54] A. Kisiel, T. Taluc, W. Broniowski, and W. Florkowski, Comput. Phys. Commun. 174, 669 (2006).

[55] L. Adamczyk et al. (STAR Collaboration), Phys. Rev. C 96, 044904 (2017).

[56] S. Bhattacharyya, D. Biswas, S. K. Ghosh, R. Ray, and P. Singha, Phys. Rev. D 101, 054002 (2020).

[57] F. Becattini, arXiv:0707.4154.

[58] A. Cayley, Quart. J. Pure Appl. Math. 23, 376 (1889). 
[59] A. Cayley, The Collected Mathematical Papers. Cambridge Library Collection (Cambridge University Press, Camdrige, UK, 2009), Vol. 13.

[60] R. L. Graham and P. Hell, Ann. Hist. Comput. 7, 43 (1985).

[61] T. H. Cormen, C. E. Leiserson, R. L. Rivest, and C. Stein, Introduction to Algorithms, 3rd ed. (MIT Press, Cambridge, MA, 2009).

[62] M. Chakraborty, S. Chowdhury, J. Chakraborty, R. Mehera, and R. K. Pal, Complex Intell. Syst. 5, 265 (2019).

[63] L. Kumar (STAR Collaboration), Nucl. Phys. A 904-905, 256c (2013).

[64] S. Das (STAR Collaboration), Nucl. Phys. A 904-905, 891c (2013).

[65] C. Adler et al. (STAR Collaboration), Phys. Rev. Lett. 89, 092301 (2002).

[66] J. Adams et al. (STAR Collaboration), Phys. Rev. Lett. 92, 182301 (2004).

[67] X. Zhu (STAR Collaboration), Acta Phys. Pol. Suppl. 5, 213 (2012).

[68] F. Zhao (STAR Collaboration), J. Phys. Conf. Ser. 509, 012085 (2014).

[69] L. Kumar (STAR Collaboration), Nucl. Phys. A 931, 1114 (2014).

[70] S. Das (STAR Collaboration), J. Phys. Conf. Ser. 509, 012066 (2014).

[71] B. I. Abelev et al. (STAR Collaboration), Phys. Rev. C 79, 034909 (2009).

[72] M. M. Aggarwal et al. (STAR Collaboration), Phys. Rev. C 83, 024901 (2011).

[73] B. I. Abelev et al. (STAR Collaboration), Phys. Rev. C 79, 064903 (2009).

[74] K. Adcox et al. (PHENIX Collaboration), Phys. Rev. Lett. 89. 092302 (2002).

[75] C. Adler et al. (STAR Collaboration), Phys. Rev. C 65, 041901 (2002).
[76] J. Adams et al. (STAR Collaboration), Phys. Rev. Lett. 98, 062301 (2007).

[77] J. Adams et al. (STAR Collaboration), Phys. Lett. B 612, 181 (2005).

[78] L. Kumar (STAR Collaboration), Central Eur. J. Phys. 10, 1274 (2012).

[79] B. Abelev et al. (ALICE Collaboration), Phys. Rev. Lett. 109, 252301 (2012).

[80] B. B. Abelev et al. (ALICE Collaboration), Phys. Rev. Lett. 111, 222301 (2013).

[81] B. B. Abelev et al. (ALICE Collaboration), Phys. Lett. B 728, 216 (2014); 734, 409(E) (2014).

[82] B. Abelev et al. (ALICE Collaboration), Phys. Rev. C 88, 044910 (2013).

[83] M. Tanabashi et al. (Particle Data Group), Phys. Rev. D 98, 030001 (2018).

[84] D. Zschiesche, S. Schramm, J. Schaffner-Bielich, H. Stoecker, and W. Greiner, Phys. Lett. B 547, 7 (2002).

[85] V. Koch, J. Phys. G 30, S41 (2004).

[86] G. Brown and M. Rho, Phys. Rep. 363, 85 (2002).

[87] R. Hagedorn, Nuovo Cimento Suppl. 3, 147 (1965).

[88] E. Witten, Phys. Rev. D 30, 272 (1984).

[89] J. Stachel, A. Andronic, P. Braun-Munzinger, and K. Redlich, J. Phys. Conf. Ser. 509, 012019 (2014).

[90] P. Huovinen and P. Petreczky, Phys. Lett. B 777, 125 (2018).

[91] P. Huovinen and P. Petreczky, PoS Confinement2018, 145 (2018).

[92] J. Steinheimer, J. Aichelin, and M. Bleicher, Phys. Rev. Lett. 110, 042501 (2013).

[93] M. Petrán, J. Letessier, V. Petráček, and J. Rafelski, Phys. Rev. C 88, 034907 (2013).

[94] F. Becattini, M. Bleicher, E. Grossi, J. Steinheimer, and R. Stock, Phys. Rev. C 90, 054907 (2014). 